\title{
Bimodal counting statistics in single-electron tunneling through a quantum dot
}

\author{
C. Fricke, ${ }^{1}$ F. Hohls, ${ }^{1}$ W. Wegscheider, ${ }^{2}$ and R. J. Haug $^{1}$ \\ ${ }^{1}$ Institut für Festkörperphysik, Leibniz Universität Hannover, Appelstraße 2, D-30167 Hannover, Germany \\ ${ }^{2}$ Angewandte und Experimentelle Physik, Universität Regensburg, D-93040 Regensburg, Germany
}

(Received 28 June 2007; published 9 October 2007)

\begin{abstract}
We explore the full counting statistics of single-electron tunneling through a quantum dot using a quantum point contact as noninvasive high bandwidth charge detector. The distribution of counted tunneling events is measured as a function of gate and source-drain voltages for several consecutive electron numbers on the quantum dot. For certain configurations, we observe super-Poissonian statistics for bias voltages at which excited states become accessible. The associated counting distributions interestingly show a bimodal characteristic. Analyzing the time dependence of the number of electron counts, we relate this to a slow switching between different electron configurations on the quantum dot.
\end{abstract}

DOI: $10.1103 /$ PhysRevB.76.155307

PACS number(s): 73.23.Hk, 72.70. $+\mathrm{m}, 73.63 . \mathrm{Kv}$

\section{INTRODUCTION}

The dynamics of electron transport through a quantum dot cannot be accessed by measurements of the average (dc) current alone. Additional information was successfully gained from measurements of the shot noise ${ }^{1-3}$ and recently even of the third moment ${ }^{4}$ of the current through the quantum dot. However, it is hard to see how such measurements could be extended to even higher moments.

Recently, an alternative approach was pointed out for semiconductor quantum dots. Besides a direct measurement of the resonant tunneling current through the dot, one can also measure the charge on the quantum dot using a nearby quantum point contact as a noninvasive and highly sensitive detector. ${ }^{5-8}$ For sufficiently low tunneling rates and high detector bandwidth, individual tunneling events onto and off the dot can be resolved. ${ }^{9,10}$ This allows a measurement of the full counting statistics for tunneling through a quantum $\operatorname{dot}^{11-13}$ and the analysis of conditional counting effects. ${ }^{14,15}$

We have implemented a measurement of single-electron counting with large bandwidth. This enables us to measure the full counting statistics for single-electron transport through a quantum dot as a function of both bias and gate voltages for a series of consecutive electron numbers on the dot. We observe well understood counting distributions for tunneling through the ground states of the quantum dot. However, interestingly, our analysis reveals also the occurrence of bimodal counting distributions for certain numbers of electrons on the dot and sufficient bias voltage. We relate this to a slow switching between two different quantum dot configurations that have distinct tunnel couplings to the leads.

\section{DEVICE AND EXPERIMENTAL TECHNIQUES}

Our device is based on a GaAs/AlGaAs heterostructure containing a two-dimensional electron system (2DES) $34 \mathrm{~nm}$ below the surface. The electron density is $n=4.59$ $\times 10^{15} \mathrm{~m}^{-2}$, the mobility is $\mu=64.3 \mathrm{~m}^{2} / \mathrm{V} \mathrm{s}$. We have used an atomic force microscope (AFM) to define the quantum dot (QD) and the quantum point contact (QPC) structure by local anodic oxidation on the surface; ${ }^{16,17}$ the 2DES below the oxidized surface is depleted and insulating areas can be written.

An AFM image of our device is presented in Fig. 1(a). The bright walls depict the insulating lines written by the AFM. The QPC (left area) is separated from the QD structure (right area) by an insulating line. The QPC can be tuned using the in-plane gate G3. The QD is coupled to source and drain via two tunneling barriers, which can be separately controlled with gates G1 and G2. These gates are also used to set the number of electrons in the QD. We use two electrically separated circuits for simultaneous conductance measurements through the QPC and the QD. The experimental setup allows us to resolve tunneling times $\tau$ as small as $30 \mu$ s. In Fig. 1(b), the time resolved current through the QPC is shown. Distinct changes in the current occur for every change of the number of electrons on the nearby QD. For example, the current drops down whenever an electron enters the dot and thus the number of electron changes from $N$ to

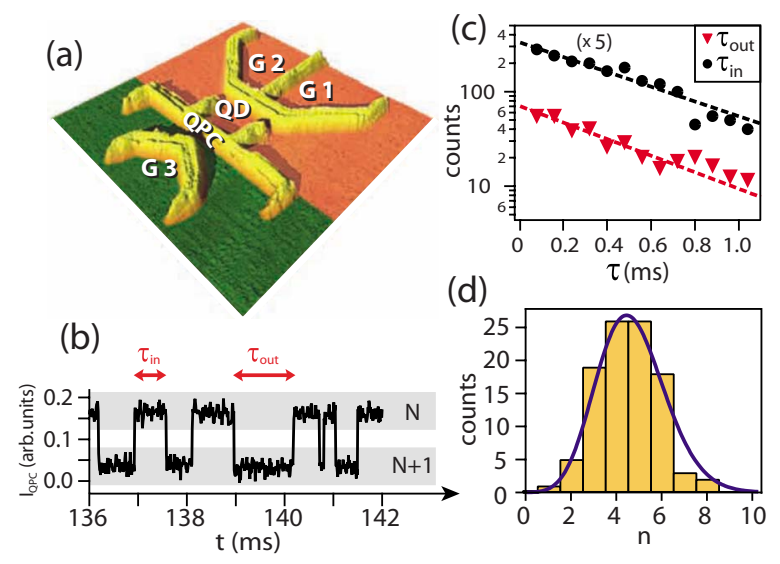

FIG. 1. (Color online) Operating principle of the device containing a QD and a QPC. (a) AFM image of the device and gate configuration. (b) Time segment of the QPC signal, tunneling times $\tau_{\text {in }}$ and $\tau_{\text {out }}$ are marked. (c) Distribution of tunneling times extracted from 100 time segments. Each time segment spans $5 \mathrm{~ms}$. The distribution for $\tau_{\text {in }}$ is shifted by a factor of 5 for clarity. (d) Distribution of tunneling events for same measurement compared to a theoretical model calculation. In total, 446 electrons passed the QD during the measurement leading to this distribution. 
$N+1$. Due to the sufficient large bias voltage of $0.2 \mathrm{mV}$, electrons are entering the QD solely from the source and are leaving the QD to the drain. In the time segment shown here, five traversing electrons can be identified. Additionally, the tunneling times $\tau_{\text {in }}$ and $\tau_{\text {out }}$ can be extracted from the QPC signal, where $\tau_{\text {in }}$ is the time until an additional electron hops from source onto the dot and $\tau_{\text {out }}$ is the time that an electron needs to leave the dot into the drain.

The statistical distributions of the tunneling times $\tau_{\text {in }}$ and $\tau_{\text {out }}$ are shown in Fig. 1(c). They follow an exponential decay, where the exponent is given by the tunneling rates $\Gamma_{\text {in }}$ and $\Gamma_{\text {out }}$, respectively. We find $\Gamma_{\text {in }}=1.7 \mathrm{kHz}$ and $\Gamma_{\text {out }}$ $=1.9 \mathrm{kHz}$. Alternatively, one can also determine these rates directly from the mean value of the tunneling times using the relation $\Gamma_{\text {in(out) }}=\left\langle\tau_{\text {in(out }}\right\rangle^{-1}$. We find a good agreement between both methods if the rates are small compared to the bandwidth of our measurement. For fast rates, we will miss a number of short transitions, which affects the mean of the tunneling times. ${ }^{18}$ Here, the analysis of the slope of the distribution yields superior results.

In this paper, we will additionally apply a further method to extract the statistical properties of the single-electron transport. The measured long time trace is divided into a large number of short segments, as depicted in Fig. 1(b), and the number of transitions $n$ from $N+1$ to $N$ electrons is counted for each segment. ${ }^{11}$ We now determine the statistical distribution of these counts $n$, as depicted in Fig. 1(d). The experimental results (bars) compare well to the theoretical model (line) calculated without free parameters from the mean of $\tau_{\text {in }}$ and $\tau_{\text {out }}{ }^{11,19}$ From the distribution, one can extract not only the mean value of $n$ but also higher moments like the Fano factor $\alpha$, which is given by the second moment of the obtained distribution divided by the mean value $\langle n\rangle$,

$$
\alpha=\frac{\left\langle(n-\langle n\rangle)^{2}\right\rangle}{\langle n\rangle} .
$$

\section{NONLINEAR TRANSPORT}

Now, we will study the statistics in detail for nonlinear transport through the QD. In Fig. 2(a), the rate of electrons per second traversing the QD as deduced from $\langle n\rangle$ is shown as a function of gate voltage and bias. Each data point represents a full measurement of the distribution of counting events and the rate is determined from the mean value $\langle n\rangle .{ }^{11}$ We clearly observe the so-called Coulomb diamonds well known from conventional transport experiments. Clear Coulomb blockade regions are found as well as discrete regions of finite current due to single-electron transport through the ground state and for large bias also through excited states of the QD. The QPC detector can also be used to determine the mean charge of the QD. ${ }^{20}$ For this, we analyzed the dc through the QPC and extracted the mean charge information. The changes in the mean charge of the QD are shown as lines in Fig. 2. The dc charge detection compares well with the results of the real-time measurement. When the mean charge of the dot changes, a distinct step in the counting rate can be seen.

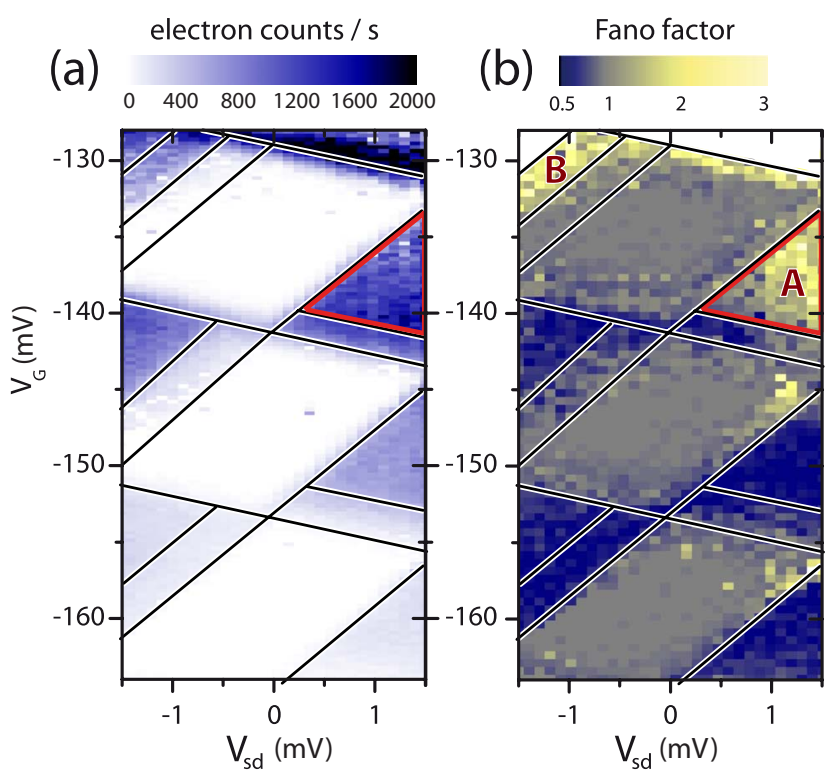

FIG. 2. (Color online) (a) Counting rate of electrons passing the $\mathrm{QD}$ as a function of gate voltage and source-drain voltage. The lines mark changes of the average QD occupation, which are deduced from the dc average of the detector signal. (b) Fano factor $\alpha$ extracted from the full counting statistics for the same range. For the white areas above $-130 \mathrm{mV}$, at least one of the tunneling rates becomes too high in order to extract a reliable counting statistic.

In Fig. 2(b), the Fano factor $\alpha$ as determined from Eq. (1) is shown for the same measurement. It turns out that $\alpha$ is between 0.5 and 1 for most of the QD configurations as one would expect for single-electron transport through the ground state of a QD. ${ }^{3}$ However, for a special area marked by the red triangle in Fig. 2 (region A), super-Poissonian noise is observed. In the marked range, not only the ground state but also excited states take part in transport. While the ground-state transport below region A shows sub-Poissonian characteristic, $\alpha$ rises dramatically when an excited state enters the transport window. A similar behavior can also be observed for the opposite transport direction at the next higher electron number (Fig. 2, region B). Below region B, the ground-state transport is strongly suppressed but when the excited state takes part in the transport, the current rises and an $\alpha>1$ is observed.

\section{BIMODAL DISTRIBUTION}

We now analyze the counting statistics for region A marked by the triangle. For finite bias but only the ground state inside the bias window (directly below region A), we find no special behavior. Applying the same procedure, as described for Fig. 1(c), we obtain tunneling rates of $\Gamma_{\text {out }}$ $=3.4 \mathrm{kHz}$ and $\Gamma_{\mathrm{in}}=2.8 \mathrm{kHz}$ for $V_{\mathrm{sd}}=1.48 \mathrm{mV}$ and $V_{G}=$ $-143 \mathrm{mV}$. However, as we cross into region A, i.e., as an excited state enters the bias window, we find a completely changed counting statistic with a bimodal distribution. A characteristic distribution is shown in Fig. 3(a). In the distribution, two clearly distinct maxima can be identified instead of the single peak structure of the usual distribution function. 

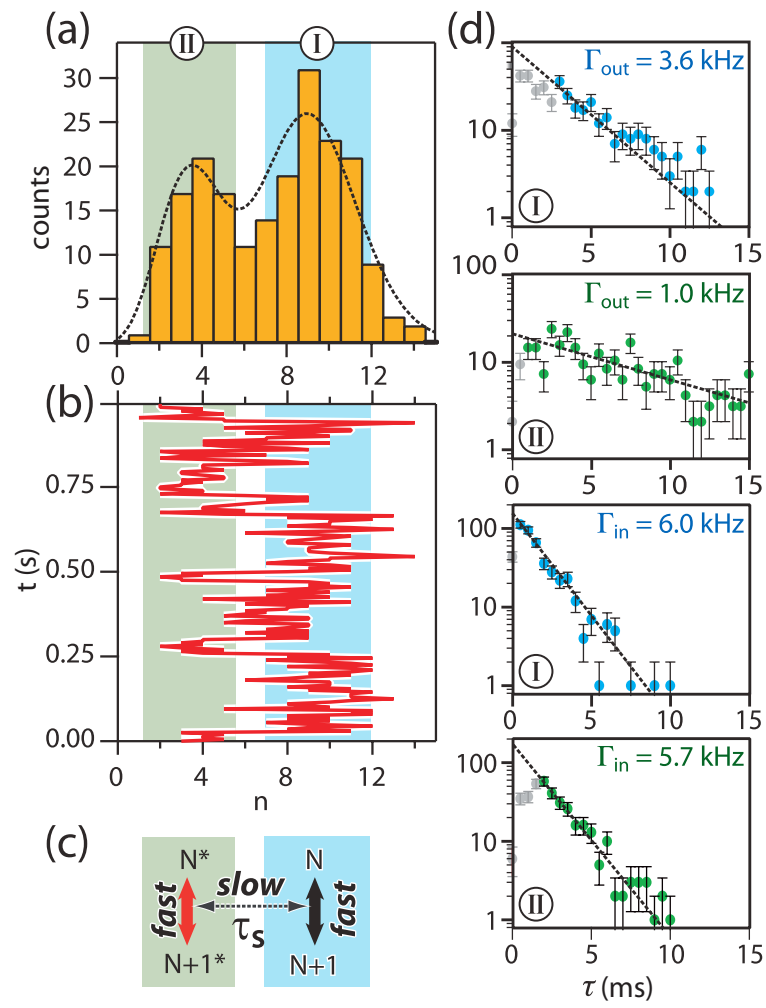

FIG. 3. (Color online) (a) Typical bimodal distribution of electrons passing through the dot for the area marked by a triangle in Fig. 2. Transport channels are marked by a number and a different background. The experimental results (bars) compare well to the basic model calculation (line). (b) Time evolution of the detected number of electrons per time segment. A switching between the two transport channels occurs. (c) Simple scheme of the experimental findings. (d) Distribution of the tunneling times $\tau_{\text {in(out) }}$ for both transport channels (dots). Short tunneling times (light gray) are suppressed due to limited bandwidth. The denoted tunneling rates $\Gamma_{\text {in(out) }}$ are extracted from the exponential fit (line).

Interestingly, one of the maxima emerges at a lower number of counts than the mean $\langle n\rangle$ observed for tunneling through the ground state before an excited state comes into play. How does the distribution relate to the rate and the Fano factor displayed in Fig. 2? Firstly, the bimodal structure leads to a significantly higher width of the distribution that results in the strong increase of the Fano factor. In contrast, the mean value does not show such a dramatic change [compare Fig. 2(a)], whereas the mean value of the bimodal distribution itself has a fairly low probability to be observed.

To study the origin of the bimodality, we now analyze the evolution of the counted electron events with time. This is shown in Fig. 3(b). Depicted is the number of electrons entering the QD in a given time segment in chronological order. By this, we can examine the temporal evolution of all time segments contained in the distribution of Fig. 3(a). As expected from the bimodal distribution, there are two separated ranges where the majority of events are accumulated. These are marked by the two darker bands in the back of Figs. 3(a) and 3(b). Moreover, a switching between these two ranges can be observed. Typically, the system stays in each of this two ranges for a time $\tau_{s}$ of 50-250 ms which is fairly long compared to the typical tunneling times $\left\langle\tau_{\text {in/out }}\right\rangle$ of about $0.2-1 \mathrm{~ms}$. For the second region showing super-Poissonian noise (Fig. 2, region B), we found the same bimodal characteristic and also a comparable switching behavior. Therefore, this bimodality seems to be a more general feature observable at different electron numbers.

Our observations can be explained by the existence of two different QD configurations. Each configuration provides a transport channel for electrons. This behavior leads to the super-Poissonian noise but differs from the situation described for multilevel quantum dots. ${ }^{13,21}$

\section{TRANSPORT CHANNELS STUDIED INDIVIDUALLY}

To acquire a deeper understanding of the ongoing processes, we analyzed the tunneling times of the two transport channels in detail. For this, we individually studied tunneling events that can be related to one of the two QD configurations.

The resulting distributions of the tunneling times $\tau_{\text {in(out) }}$ are shown in Fig. 3(d) separately for configurations I (first and third graphs) and II (second and fourth graphs). All sets of data were fitted by exponential decay to extract the tunneling rates. While the tunneling barriers were roughly symmetric for the ground state as deduced from similar values of $\Gamma_{\text {in }}$ and $\Gamma_{\text {out }}$ (see Fig. 1 and the values given for $V_{\text {sd }}$ $=1.48 \mathrm{mV}$ ), they get clearly asymmetric for the analyzed situation.

When the first excited state enters the transport window, the tunneling rate $\Gamma_{\text {in }}$ has risen rather evenly for both configurations I $(6.0 \mathrm{kHz})$ and II $(5.7 \mathrm{kHz})$. Both configurations seem to differ only slightly in the coupling to the source lead. For the drain lead, the result is different. $\Gamma_{\text {out }}$ for configuration I $(3.6 \mathrm{kHz})$ is still comparable to the ground state. In contrast, configuration II shows a tunneling rate $\Gamma_{\text {out }}$ of only $1 \mathrm{kHz}$ that is far less than the outgoing tunneling rate of the ground-state transport. When the QD is in configuration II, tunneling off the dot is suppressed as the QD seems to couple less efficiently to the drain lead.

\section{TWO CHANNEL MODEL}

We can model the strong bimodality of the counting distribution assuming two independent transport channels. Starting from the full counting statistics theory ${ }^{12}$ for single state transport through a QD, we combine two distribution functions to model our experimental results. The probability to measure a number $n$ of traversing electrons is given by

$$
P(n)=q \int_{-\pi}^{\pi} e^{-S_{1}(\chi)-n \chi \frac{d \chi}{2 \pi}}+(1-q) \int_{-\pi}^{\pi} e^{-S_{2}(\xi)-n \xi} \frac{d \xi}{2 \pi},
$$

where $S_{1}$ and $S_{2}$ are the generating functions for the separate transport channels and $q$ is the probability to detect transport through channel I. The generating function of a single level QD has been calculated for unidirectional tunneling as observed at sufficiently large bias, ${ }^{19}$ 


$$
\frac{S(x)}{t_{0}}=\left(\Gamma_{\text {in }}+\Gamma_{\text {out }}-\sqrt{\left(\Gamma_{\text {in }}-\Gamma_{\text {out }}\right)^{2}+4 \Gamma_{\text {in }} \Gamma_{\text {out }} e^{-i x}}\right) .
$$

We use the tunneling rates extracted individually for the two QD configurations [Fig. 3(d)], which leaves only $q$ as the remaining free parameter. The resulting distribution function shown in Fig. 3(a) (dashed line) was received for $q=0.51$. The good agreement of model and experimental data further confirms the idea of a switching between two dot configurations, where the dynamics of each configuration individually is described by a single pair of tunneling rates $\Gamma_{\text {in }}$ and $\Gamma_{\text {out }}$.

The most likely nature of the different QD configurations is the excitation of an electron into a nonequilibrium single particle state that couples only weakly to source and drain. If the ground-state transition is given by $(N) \rightarrow(N+1) \rightarrow(N)$, we could relate this to configuration I. Thus, the change of configuration I to II would involve a process $(N) \rightarrow\left(N+1^{*}\right)$ $\rightarrow\left(N^{*}\right)$ with $(N)$ and $(N+1)$ the ground states for $N$ and $N$ +1 electrons and $\left(N^{*}\right)$ an excited state. If the lowest lying free single-particle state is only weakly coupled, we can carry a single-electron current in the cycle $\left(N^{*}\right) \rightarrow\left(N+1^{*}\right)$ $\rightarrow\left(N^{*}\right)$ [compare Fig. 3(c)] until the less probable $\left(N+1^{*}\right)$ $\rightarrow(N)$ transition reinstates configuration I (ground-state transition).

The two configurations can arise from two origins: spin or charge. We find that the bimodality only appears when excited states with a significant excitation energy of $0.3 \mathrm{meV}$ are accessible. We assume that the occurrence of two transport channels is caused by two different charge configurations. A change of the spin configuration should mainly change the tunneling rate into the dot due to a change of spin selection rule and should have only small effect on the outgoing rate. In contrast, we observe for both configurations roughly the same tunneling rate for electrons entering the QD, while a significant difference occurs in the outgoing rate. The experimental results therefore favor a charge-type effect.

So far we focused on the two dot configurations and the transport cycles $(N) \rightarrow(N+1) \rightarrow(N)$ and $\left(N^{*}\right) \rightarrow\left(N+1^{*}\right)$ $\rightarrow\left(N^{*}\right)$. For the characterization of the less probable $(N$ $\left.+1^{*}\right) \rightarrow(N)$ or $(N) \rightarrow\left(N+1^{*}\right)$ transition, we have to analyze the switching process in more detail.

For this, we extract the switching events from the experimental data. The time the dot stays in configuration $I$ is named $\tau_{\mathrm{I}}$ and the occupation time of configuration II is named $\tau_{\text {II }}$. To extract the switching times, we have analyzed many time segments like the one shown in the lower part of Fig. 3(b). For each segment, the number of switching processes and the times $\tau_{\mathrm{I}}$ and $\tau_{\mathrm{II}}$ between the switching events are determined. In Fig. 4(a), the statistics of the observed switching events is shown. We find the center of the distribution at $\left\langle n_{\text {switch }}\right\rangle=3$ switching events per second. The distribution shows a width of about 2.5, which corresponds approximately to $50 \%$ of the width of a Poissonian distribution. Thus, the switching is a sub-Poissonian process.

The statistical distributions of the switching times $\tau_{\mathrm{I}}$ and $\tau_{\text {II }}$ are shown in Fig. 4(b). They follow an exponential decay, where the exponent is given by the inverse value of the mean
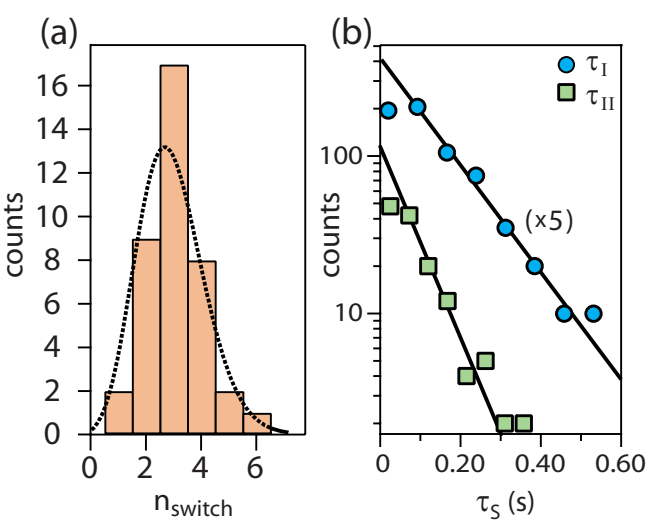

FIG. 4. (Color online) (a) Distribution of switching events during a $1 \mathrm{~s}$ time interval. The experimental data (bars) compare well to the theoretical model of a two level system (dotted line). (b) Statistical distribution of the switching times $\tau_{\text {high }}$ and $\tau_{\text {low }}$. From an exponential fit, one can determine the average occupation time of the two dot states.

switching times. From the exponential fit (line), we can extract the mean value of the switching times $\left\langle\tau_{\mathrm{I}}\right\rangle=130 \mathrm{~ms}$ and $\left\langle\tau_{\mathrm{II}}\right\rangle=75 \mathrm{~ms}$. The mean values of $\tau_{\mathrm{I}(\mathrm{II})}$ are equivalent to the average occupation time of the two dot configurations.

The switching process itself can be described as a two level system with two transition rates $\Gamma_{\mathrm{I}}$ and $\Gamma_{\mathrm{II}}$. To calculate the theoretical distribution expected for such a system, ${ }^{19}$ we need to evaluate the switching rates, which can be obtained using the relation $\Gamma_{\mathrm{I}(\mathrm{II})}=\left\langle\tau_{\mathrm{I}(\mathrm{II})}\right\rangle^{-1}$. In Fig. 4(a), the resulting curve (line) is compared to the experimental result (bars). The model of a sub-Poissonian two state system describes the experimental data very well.

\section{CONCLUSION}

To conclude, we have shown full counting statistics of single-electron tunneling through a quantum dot using a quantum point contact as a noninvasive high bandwidth charge detector. We observe super-Poissonian noise for certain QD configurations where excited states take part in electron transport. For these configurations, a clear bimodality of the electron counting distribution occurs. We have analyzed the bimodal distribution in detail and find a slow switching behavior in the dot transport. We have also analyzed the tunneling times for both configurations and presented a model. The good agreement of model and experimental results confirms the presumption of two independent transport channels. The switching between two independent transport channels can be explained by the existence of two different QD configurations. Its statistical properties agree well with a theoretical two state model. Finally, we have shown that the observed super-Poissonian transport arises from a subPoissonian switching process between two dot configurations each showing a sub-Poissonian transport characteristic.

\section{ACKNOWLEDGMENT}

This work has been supported by BMBF in the framework of nanoQUIT. 
${ }^{1}$ Y. M. Blanter and M. Büttiker, Phys. Rep. 336, 1 (2000).

${ }^{2}$ A. Nauen, I. Hapke-Wurst, F. Hohls, U. Zeitler, R. J. Haug, and K. Pierz, Phys. Rev. B 66, 161303(R) (2002).

${ }^{3}$ A. Nauen, F. Hohls, N. Maire, K. Pierz, and R. J. Haug, Phys. Rev. B 70, 033305 (2004).

${ }^{4}$ B. Reulet, J. Senzier, and D. E. Prober, Phys. Rev. Lett. 91, 196601 (2003).

${ }^{5}$ M. Field, C. G. Smith, M. Pepper, D. A. Ritchie, J. E. F. Frost, G. A. C. Jones, and D. G. Hasko, Phys. Rev. Lett. 70, 1311 (1993).

${ }^{6}$ J. M. Elzerman, R. Hanson, J. S. Greidanus, L. H. Willems van Beveren, S. De Franceschi, L. M. K. Vandersypen, S. Tarucha, and L. P. Kouwenhoven, Phys. Rev. B 67, 161308(R) (2003).

${ }^{7}$ R. Schleser, E. Ruh, T. Ihn, K. Ensslin, D. C. Driscoll, and A. C. Gossard, Appl. Phys. Lett. 85, 2005 (2004).

${ }^{8}$ C. Fricke, M. C. Rogge, B. Harke, M. Reinwald, W. Wegscheider, F. Hohls, and R. J. Haug, Phys. Rev. B 72, 193302 (2005).

${ }^{9}$ J. M. Elzerman, R. Hanson, L. H. Willems van Beveren, B. Witkamp, L. M. K. Vandersypen, and L. P. Kouwenhoven, Nature (London) 430, 431 (2004).

${ }^{10}$ T. Fujisawa, T. Hayashi, R. Tomita, and Y. Hirayama, Science 312, 1634 (2006).

${ }^{11}$ S. Gustavsson, R. Leturcq, B. Simovic, R. Schleser, T. Ihn, P.
Studerus, K. Ensslin, D. C. Driscoll, and A. C. Gossard, Phys. Rev. Lett. 96, 076605 (2006).

${ }^{12}$ L. S. Levitov, H. Lee, and G. B. Lesovik, J. Math. Phys. 37, 4845 (1996).

${ }^{13}$ S. Gustavsson, R. Leturcq, B. Simovic, R. Schleser, P. Studerus, T. Ihn, K. Ensslin, D. C. Driscoll, and A. C. Gossard, Phys. Rev. B 74, 195305 (2006).

${ }^{14}$ A. N. Jordan and E. V. Sukhorukov, Phys. Rev. Lett. 93, 260604 (2004).

${ }^{15}$ E. V. Sukhorukov, A. N. Jordan, S. Gustavsson, R. Leturcq, T. Ihn, and K. Ensslin, Nat. Phys. 3, 243 (2007).

${ }^{16}$ R. Held, T. Vancura, T. Heinzel, K. Ensslin, M. Holland, and W. Wegscheider, Appl. Phys. Lett. 73, 262 (1998).

${ }^{17}$ U. F. Keyser, H. W. Schumacher, U. Zeitler, R. J. Haug, and K. Eberl Appl. Phys. Lett. 76, 457 (2000).

${ }^{18}$ O. Naaman and J. Aumentado, Phys. Rev. Lett. 96, 100201 (2006).

${ }^{19}$ D. A. Bagrets and Yu. V. Nazarov, Phys. Rev. B 67, 085316 (2003).

${ }^{20}$ M. C. Rogge, B. Harke, C. Fricke, F. Hohls, M. Reinwald, W. Wegscheider, and R. J. Haug, Phys. Rev. B 72, 233402 (2005).

${ }^{21}$ W. Belzig, Phys. Rev. B 71, 161301(R) (2005). 